\title{
ОСОБЕННОСТИ СТРУКТУРНО-ФАЗОВЫХ ПРЕВРАЩЕНИЙ ПРИ ОТЖИГЕ ПРОТОНООБМЕННЫХ СЛОЕВ В КРИСТАЛЛАХ НИОБАТА ЛИТИЯ
}

\author{
2017 () С. С. Мушинский, И. В. Петухов, М. А. Пермякова, В. И. Кичигин, \\ Л. Н. Малинина, А. Б. Волынцев \\ Пермский государственный национальный исследовательский университет, \\ ул. Букирева, 15, 614990 Пермь, Россия \\ e-mail: Petukhov-309@yandex.ru
}

Поступила в редакцию 04.08.2017

\begin{abstract}
Аннотация. С использованием методов оптической микроскопии в поляризованном свете, модовой спектроскопии, ИК-спектроскопии и рентгеноструктурного анализа подтверждена последовательность фазовых превращений, происходящих при отжиге протонообменного слоя на X-срезе кристалла ниобата лития: $\beta_{2}-$, $\beta_{1}$-фазы $\rightarrow \kappa_{2}$-фаза $\rightarrow \kappa_{1}$-фаза $\rightarrow \alpha$-фаза. Установлено, что при превращении $\kappa_{2} \rightarrow \kappa_{1}$ образуются частицы $\kappa_{1}$-фазы (в виде упорядоченных структур), а не слой этой фазы.
\end{abstract}

Ключевые слова: ниобат лития, Х-срез, протонный обмен, отжиг, фазовые превращения.

\section{ВВЕДЕНИЕ}

Кристаллы ниобата лития широко применяются в интегральной оптике для изготовления интегрально-оптических фазовых модуляторов [1]. Для этого в поверхностных слоях кристалла $\mathrm{LiNbO}_{3}$ методом протонного обмена (ПО) формируются волноводы с отличающимся от объемного значения показателем преломления. Протонный обмен проводится в расплаве бензойной кислоты, при этом часть ионов лития в кристалле замещается на ионы водорода. Для получения волноводов со стабильными характеристиками и низкими оптическими потерями проводится отжиг ПО-слоев при температуpax $330-400{ }^{\circ} \mathrm{C}$.

В зависимости от условий протонного обмена и отжига возможно образование до 7 различных протонообменных фаз твердого раствора $\mathrm{H}_{\mathrm{x}} \mathrm{Li}_{1-\mathrm{x}} \mathrm{NbO}_{3}$ [2]. Прямым низкотемпературным протонным обменом могут быть получены оптические волноводы, содержащие фазы $\beta_{1}, \beta_{2}$ и $\beta_{3}$. Фаза $\beta_{1}$ может самостоятельно присутствовать на поверхности пластин, тогда как $\beta_{2}$ - и $\beta_{3}$-фазы могут существовать только вместе с $\beta_{1}$ [2].

Фазовый состав ПО-волноводов, несомненно, влияет на их оптические [1] и электрооптические [3] свойства. Одинаковым изменениям показателя преломления $\Delta n_{e}$ может соответствовать раз- личный фазовый состав ПО-области и, как следствие, различные оптические характеристики волновода [1].

Большое значение имеет последовательность и кинетика фазовых превращений, происходящих при отжиге ПО-слоев, поскольку отжиг является одной из основных технологических операций при изготовлении стабильных ПО-волноводов с хорошими электрооптическими свойствами и низкими оптическими потерями [4]. Фазовые превращения при отжиге были представлены следующим образом [2]. Кратковременный отжиг при температуpe $T<340{ }^{\circ} \mathrm{C}$ приводит к фазовому превращению $\beta_{2} \rightarrow \beta_{1}$. Отжиг, проводимый после ПО, позволяет формировать новые фазы $\kappa_{1}$ и $\kappa_{2}$, которые имеют низкотемпературные $\left(\kappa_{i}^{\mathrm{LT}}\right)$ и высокотемпературные $\left(\kappa_{\mathrm{i}}^{\mathrm{HT}}\right)$ модификации. Низкотемпературные модификации формируются отжигом при температурах $T<340{ }^{\circ} \mathrm{C}$, а высокотемпературные - при $T>400{ }^{\circ} \mathrm{C}$. При отжиге возможно образование четырехслойной структуры с еще не полностью исчезнувшей с поверхности фазой $\beta_{1}$ и фазами $\kappa_{2}, \kappa_{1}$ и $\alpha$, последовательно расположенными в виде отдельных слоев. Дальнейший отжиг при $T<340{ }^{\circ} \mathrm{C}$ приводит к последовательному исчезновению фаз $\beta_{1}, \kappa_{2}^{\mathrm{LT}}$ и $\kappa_{1}^{\mathrm{LT}}$, и в конечном итоге в волноводе остается только фаза $\alpha$. 
В отличие от [2], в работе [5] для не полностью отожженного ПО-волновода предлагалась модель ПО-слоя, содержащая две фазы ( $\beta$ и $\alpha)$, причем ПО-область представляет собой не два последовательных слоя, а слой $\alpha$-фазы, в котором определенным образом распределены включения $\beta$-фазы.

В работе [6] изучена кинетика фазовых превращений в протонированном ниобате лития при отжиге с использованием ИК-спектроскопии. Обнаруживались только две фазы ( $\beta$ и $\alpha)$; за время отжига 24 ч при $350{ }^{\circ} \mathrm{C}$ достигалось практически полное превращение $\beta \rightarrow \alpha$, если продолжительность ПО в бензойной кислоте при $200{ }^{\circ} \mathrm{C}$ не превышала 4 ч.

Таким образом, необходимы дальнейшие исследования кинетики фазовых превращений в $\mathrm{H}: \mathrm{LiNbO}_{3}$ волноводах при отжиге, особенно на завершающих стадиях перехода в $\alpha$-фазу.

В работе [7] с использованием оптической микроскопии и модовой спектроскопии была изучена последовательность и кинетика фазовых превращений при отжиге протонообменных слоев на $\mathrm{X}$-срезе кристалла ниобата лития. Цель данной работы - изучить изотермические фазовые превращения в протонообменных слоях на X-срезе кристаллов ниобата лития и подтвердить образование тех или иных фаз в процессе отжига методами рентгеноструктурного анализа и ИК-спектроскопии.

\section{ЭКСПЕРИМЕНТАЛЬНАЯ ЧАСТЬ}

Для проведения исследований использовался X-срез кристалла ниобата лития конгруэнтного состава производства Crystal Technology. Рабочими образцами служили пластины толщиной 1 мм размером $15 \times 10$ мм, вырезанные из вейфера диаметром 76 мм.

Протонный обмен проводился в закрытом циркониевом реакторе в расплаве бензойной кислоты (ч. д. а.) при температуре $175^{\circ} \mathrm{C}$ в течение 2 ч. Отжиг образцов проводился последовательно с шагом 1 ч при температуре $330^{\circ} \mathrm{C}$. Суммарная продолжительность отжига составляла 12 ч.

Для исследуемых планарных волноводов определяли профили приращения показателя преломления необыкновенного луча $\Delta n_{e}(x)$ и значения $\Delta n_{e}(0)$ на поверхности волноводного слоя. Для определения $\Delta n_{e}(x)$ и $\Delta n_{e}(0)$ на планарных волноводах использовали метод модовой спектроскопии. С помощью призменного ввода измеряли эффективные показатели преломления волноводных мод на длине волны $\lambda=0.633$ мкм. Профиль $\Delta n_{e}(x)$ по глубине волноводного слоя восстанавливался с помощью обратного метода Вентцеля-КрамерсаБриллюэна [8].

Для визуализации структуры ПО-слоев использовали метод оптической микроскопии (Olympus ВХ 61) в поляризованном свете с применением методики светлого и темного полей.

Рентгенографические исследования образцов ниобата лития проводились на рентгеновском двухкристальном дифрактометре ДРОН-УМ1 в излучении кобальтового анода с использованием длины волны $\lambda_{\beta}=1.62073 \AA \AA$. Регистрировали $\theta / 2 \theta$ кривые. Величина деформации $\varepsilon_{33}$ определялась непосредственно из $\theta / 2 \theta$ кривых измерением углового расстояния $\Delta \theta$ между положением максимума дифракционного отражения от подложки ниобата лития и максимумом дифракционного отражения от соответствующей фазы $\mathrm{H}_{x} \mathrm{Li}_{1-x} \mathrm{NbO}_{3}$ в соответствии с формулой Вульфа-Брэгга в дифференциальной форме:

$$
\varepsilon_{33}=-\Delta \theta \operatorname{ctg} \theta
$$

где $\theta$ - Брэгговский угол отражения, ассоциированный с рассматриваемым семейством кристаллических плоскостей.

Образцы ниобата лития с планарными ПО-волноводами также исследовали методом ИК-спектроскопии с помощью спектрофотометра Bruker Spectrum Two в частотном диапазоне $400-6000 \mathrm{~cm}^{-1}$ с разрешением $<1 \mathrm{~cm}^{-1}$.

\section{РЕЗУЛЬТАТЫ И ОБСУЖДЕНИЕ}

Непосредственно после протонного обмена формируется одномодовый волновод, и определить значение $\Delta n_{e}(0)$ и глубину волноводного слоя с помощью обычной процедуры не представляется возможным. После отжига в течение 1 ч значение $\Delta n_{e}(0)$, как следует из структурно-фазовой диаграммы [1], соответствует низкотемпературной $\kappa_{2}$-фазе (рис. 1). Вероятно, уже в течение первого часа отжига $\beta_{1}$ - и $\beta_{2}$-фазы, образовавшиеся в результате протонного обмена, превращаются в $\kappa_{2}$-фазу.

Последующий отжиг приводит к дальнейшему снижению значения $\Delta n_{e}(0)$ и увеличению глубины волноводного слоя (рис. 1). Следует отметить, что в течение 2 и 3 ч отжига приращение показателя преломления изменяется незначительно, а его величины имеют промежуточные значения между наименьшим значением для $\kappa_{2}$-фазы и наибольшим значением для $\kappa_{1}$-фазы. Возникновение горизонтальной площадки, вероятно, означает, что в те- 
чение 2 и 3-го часа отжига происходит переход из $\kappa_{2}$-фазы в $\kappa_{1}$-фазу.

Дальнейший отжиг (вплоть до $t \leq 7$ ч) вызывает более быстрое уменьшение приращения показателя преломления и увеличение глубины волноводного слоя (рис. 1). Можно предположить, что при этом происходит постепенное превращение $\kappa_{1}$-фазы в $\alpha$-фазу.

При продолжительности отжига 8 ч и более спад приращения показателя преломления замедляется, замедляется и увеличение глубины волноводного слоя (рис. 1). Значения $\Delta n_{e}(0)$ могут указывать на дальнейшее превращение $\kappa_{1}$-фазы в $\alpha$-фазу. При продолжительности отжига 11 и 12 ч, исходя из величины $\Delta n_{e}(0)[1]$, можно утверждать, что ПОслой состоит только из $\alpha$-фазы. Уменьшение $\Delta n_{e}(0)$ и увеличение глубины волноводного слоя обусловлено диффузией протонов в объем кристалла ниобата лития.

Исследования поверхности образцов ниобата лития в поляризованном свете методом темнопольной микроскопии, в целом, согласуются с результатами модовой спектроскопии. После 1 ч отжига в протонированном слое появляются мелкие частицы другой фазы - это в матрице, состоящей из $\kappa_{2}$-фазы, образуются частицы к -фазы (рис. $2 a$ ). При времени отжига 2 ч количество включений $\kappa_{1}$-фазы увеличивается (рис. $2 b$ ). Дальнейший отжиг (3 ч) приводит к превращению значительной доли $\kappa_{2}$-фазы в $\kappa_{1}$-фазу (рис. 2c). Частицы $\kappa_{1}$-фазы образуют модулированную структуру, располагаясь вдоль определенных кристаллографических направлений.

Далее начинается более медленный процесс превращения $\kappa_{1}$-фазы в $\alpha$-фазу. После 5 ч отжига на микрофотографиях видны темные прожилки $\alpha$-фазы (рис. $2 d$ ), доля $\alpha$-фазы по мере увеличения продолжительности отжига (7 ч) увеличивается (рис. 2e). После 9 ч отжига остатки к -фазы наблюдаются на микрофотографиях в виде единичных светлых включений (рис. 2f).

Однако даже после полного превращения $\kappa_{1}$ фазы в $\alpha$-фазу, возможно, некоторые повышенные количества протонов, а также другие дефекты структуры на ранее существовавших границах раздела между $\kappa_{1}$-фазой и $\alpha$-фазой, декорируют эти границы. По этой причине бывшие границы между фазами просматриваются на светлопольных изображениях в поляризованном свете (рис. 3). Полностью однородной структура становится лишь после 12 ч отжига.

После протонного обмена, происходящего в те-
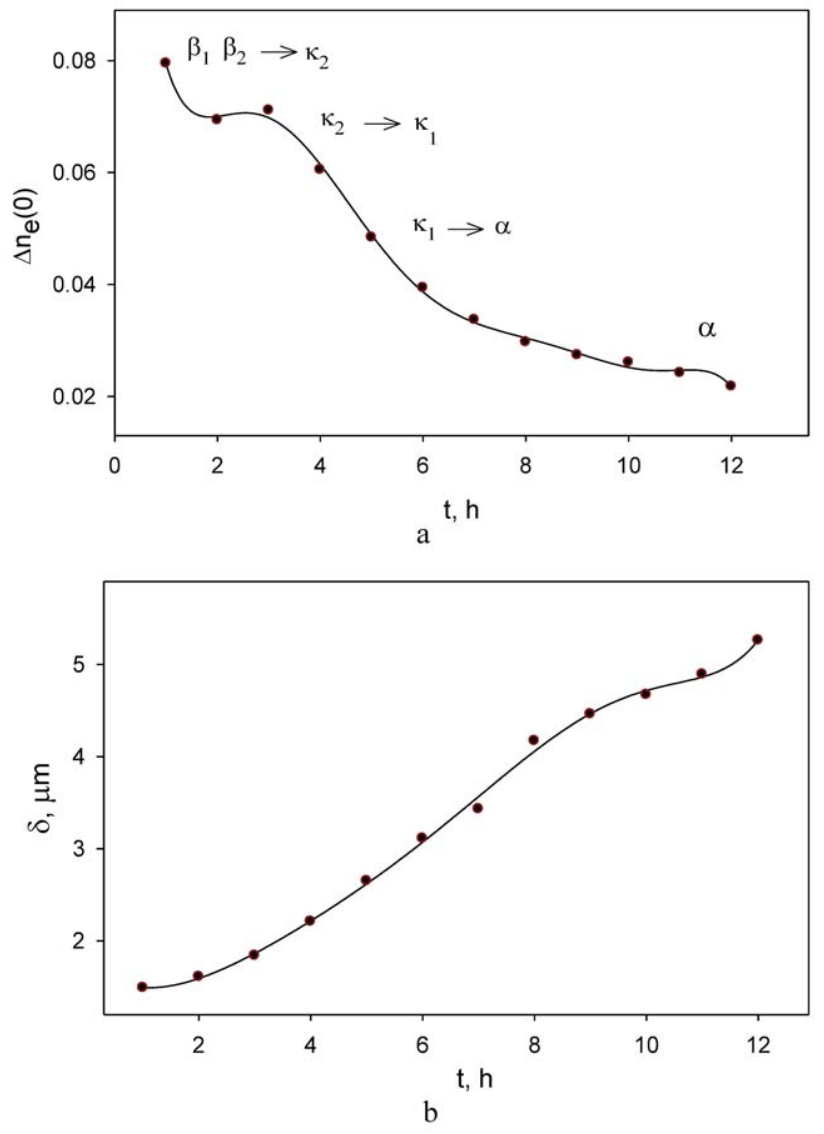

Рис. 1. Зависимость $\Delta n_{e}(0)(a)$ и глубины волноводного слоя $(b)$ от продолжительности отжига

[Fig. 1. Dependence of $\Delta n_{e}(0)(a)$ and guide layer depth (b) on annealing duration]

чение 2 ч в расплаве бензойной кислоты при температуре $175^{\circ} \mathrm{C}$, на поверхности ниобата лития формируются два тонких последовательных слоя $\beta_{1}$ - и $\beta_{2}$-фаз. Это следует из $\theta / 2 \theta$ кривой, на которой просматриваются два слабых пика, отвечающих фазам $\beta_{1}, \beta_{2}$, значения регистрируемых деформаций $\varepsilon_{33}$ соответствуют $\beta_{1}$ - и $\beta_{2}$-фазам на структурно-фазовой диаграмме [1].

При съемке каждого образца регистрировалось две $\theta / 2 \theta$ кривых разных порядков отражения. Различие между этими кривыми заключается в том, что линия (110), регистрируемая при меньших углах падения на образец, в большей мере несет информацию о состоянии поверхностных слоев исследуемого образца. Отражение с индексами (220), в силу того, что рентгеновское излучение проникает в более глубокие слои ниобата лития, указывает на фазовый состав этих слоев ниобата лития.

Отжиг в течение 1 ч приводит к появлению пика с максимумом, соответствующим 


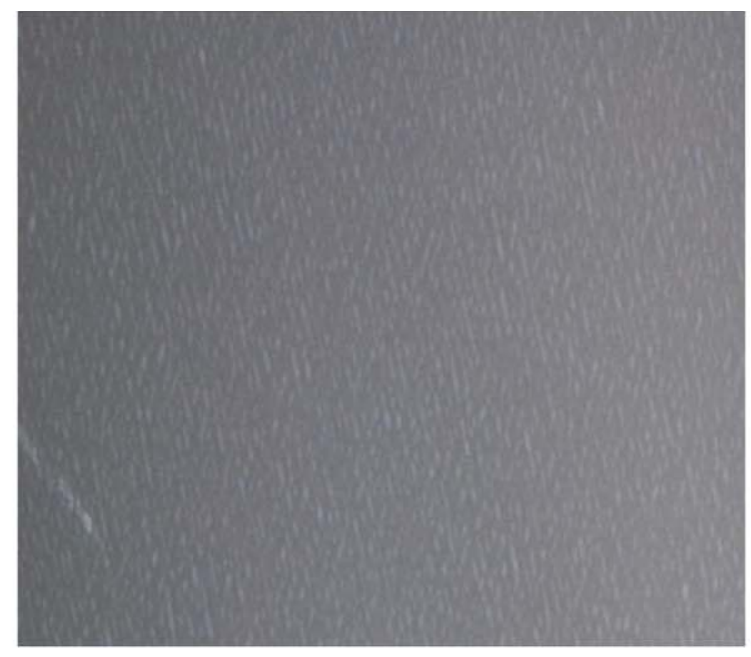

a

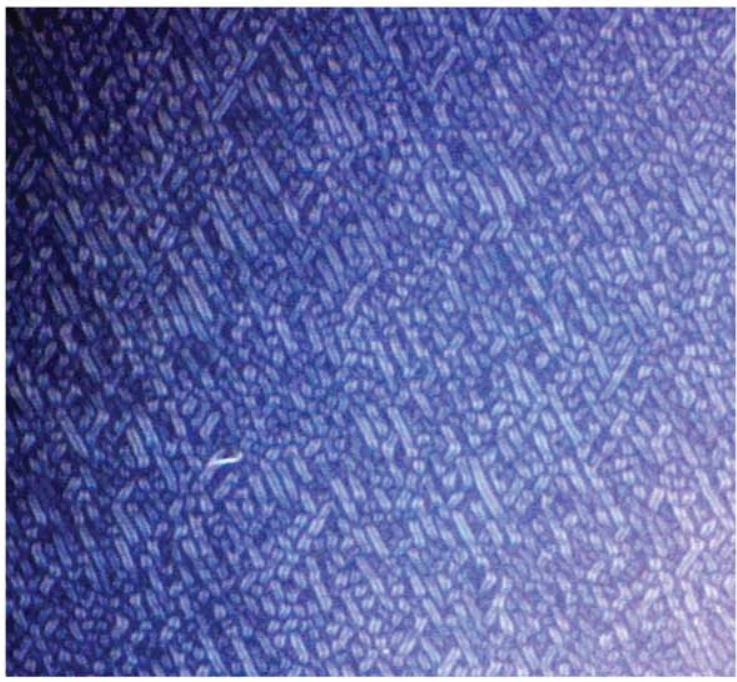

c

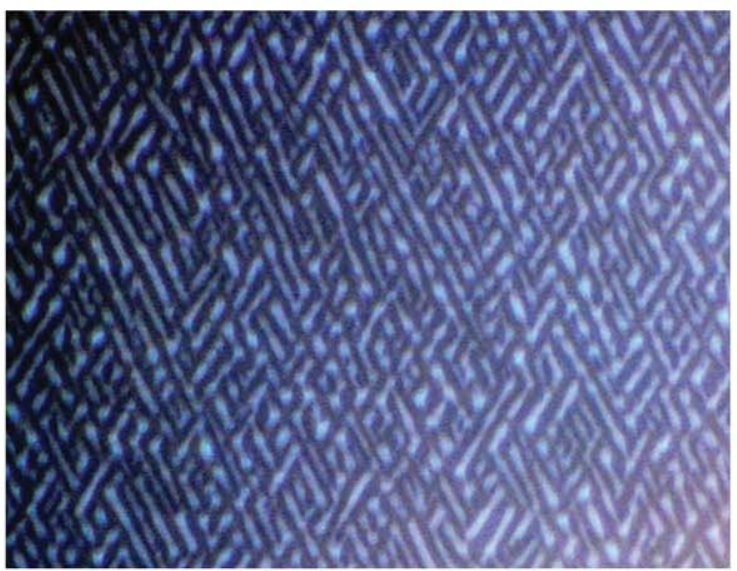

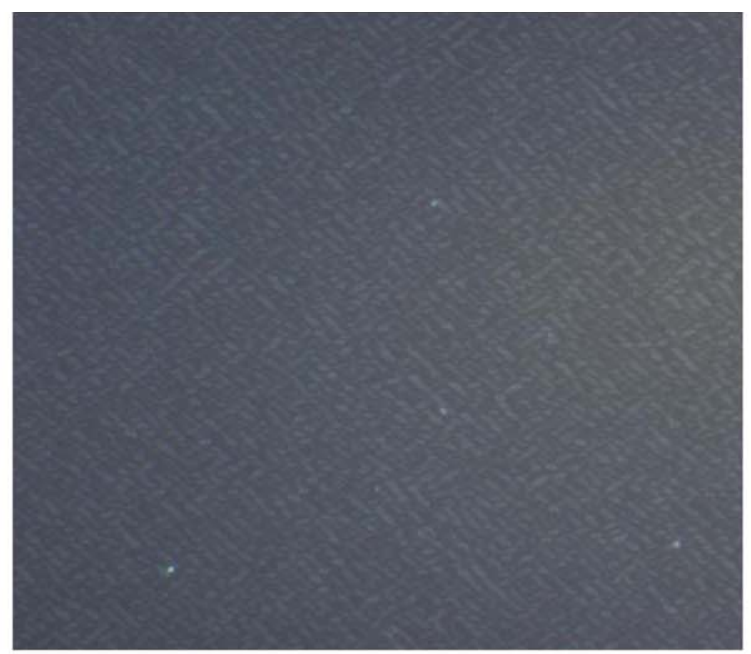

$\mathrm{b}$

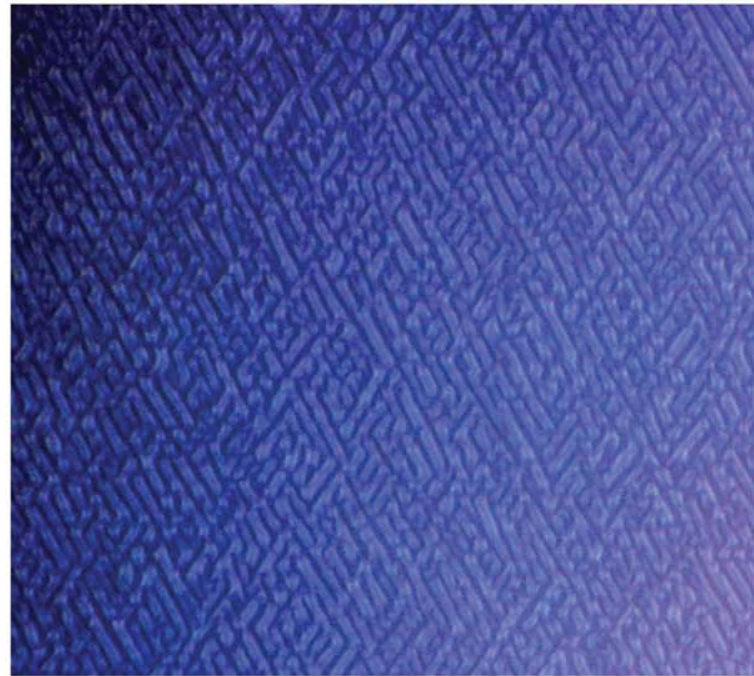

d

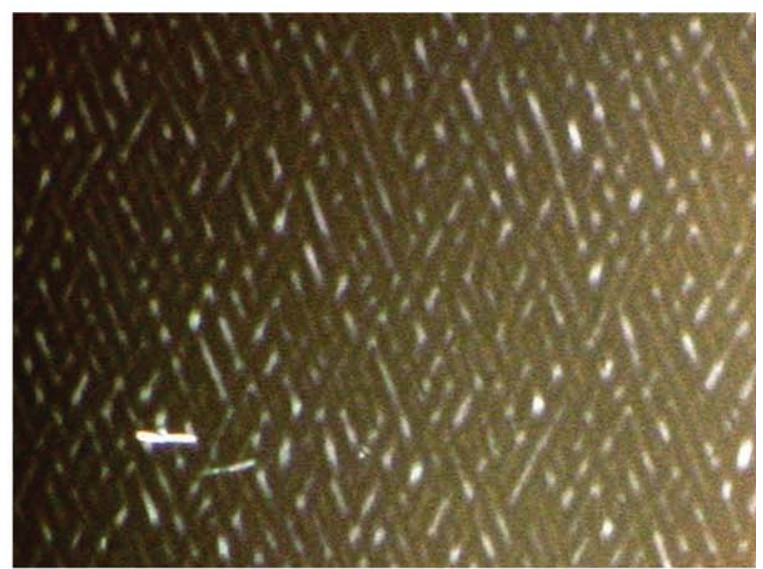

f

Рис. 2. Микроструктура поверхности протонированных образцов ниобата лития, продолжительность отжига, ч: $a-1, b-2, c-3, d-5, e-7, f-9$. Увеличение $\times 1000$

[Fig. 2. Microstructure of the surface of protonated samples of lithium niobate. Annealing duration, h: $a-1, b-2, c-3, d-5, e-7, f-9$. Magnification $\times 1000]$ 


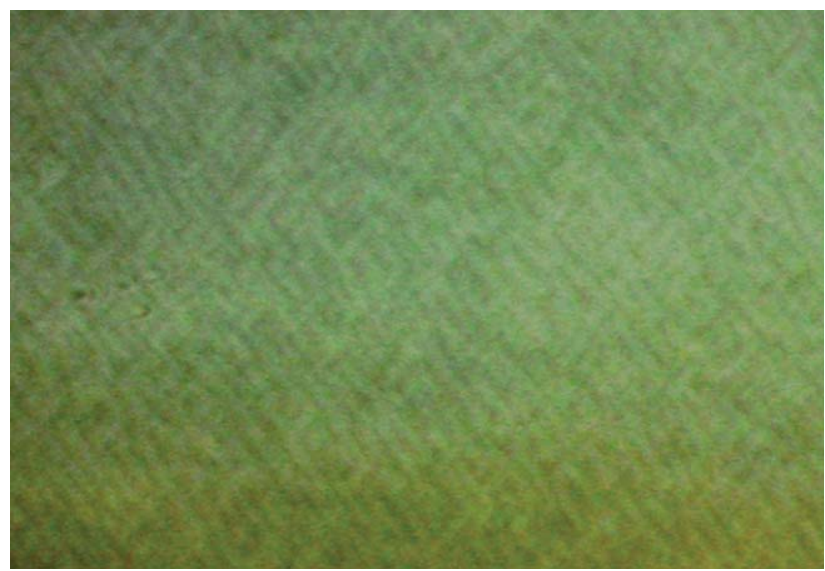

Рис. 3. Светлопольное изображение поверхности протонированного образца ниобата лития, продолжительность отжига -11 ч. Увеличение $\times 1000$

[Fig. 3. Bright-field image of the surface of protonated sample of lithium niobate annealed for $11 \mathrm{~h}$. Magnification $\times 1000$ ]

$\varepsilon_{33}=4.7 \cdot 10^{-3}$ (рис. $\left.4 a\right)$. В соответствии со структурно-фазовой диаграммой, а также результатами модовой спектроскопии, в протонообменном слое присутствует низкотемпературная $\kappa_{2}$-фаза. Пик ниобата лития является асимметричным. Это связано с тем, что на пик, соответствующий ниобату лития, накладывается отражение от низкотемпературной $\kappa_{1}$-фазы. Значения $\varepsilon_{33} \kappa_{1}$-фазы находятся в интервале $(4 \div 12) \cdot 10^{-4}$, что достаточно близко к пику ниобата лития [1]. Асимметричность данного пика лучше проявляется при регистрации более высокого порядка отражения (рис. 4a) - линии (220).

Поскольку напряжения и деформации, соответствующие $\alpha$-фазе $\left(\varepsilon_{33}<1.5 \cdot 10^{-4}\right)$, значительно меньше по сравнению с $\kappa_{1}$-фазой, разделить пики, соответствующие непосредственно ниобату лития и $\alpha$-фазе, не представляется возможным.
Увеличение продолжительности отжига до 2 ч приводит к уменьшению интенсивности пика, соответствующего $\kappa_{2}$-фазе, и дальнейшему искажению пика ниобата лития вследствие увеличения содержания $\kappa_{1}$-фазы (рис. $4 b$ ). Положение максимума пика $\kappa_{2}$-фазы практически не изменяется и соответствует деформации $\varepsilon_{33}=4.5 \cdot 10^{-3}$. Из анализа отражений (220) следует, что в более глубоких слоях уменьшается доля $\kappa_{2}$-фазы и увеличивается доля $\kappa_{1}$-фазы (рис. $4 b$ ).

Отжиг в течение 4 ч приводит почти к полному исчезновению линии $\kappa_{2}$-фазы. На ее присутствие может указывать слабый максимум, которому соответствует значение $\varepsilon_{33}=3.9 \cdot 10^{-3}$ (рис. $4 c$ ).

На $\theta / 2 \theta$ кривой второго порядка отражения данный максимум совсем отсутствует, тогда как интенсивность пика, соответствующего $\kappa_{1}$-фазе увеличивается (рис. $4 c$ ).

На $\theta / 2 \theta$ кривых, полученных после отжига в течение 6 и 9 ч, регистрируется только один асимметричный пик (рис. 4d), асимметричность которого обусловлена присутствием $\kappa_{1}$-фазы.

Для количественной оценки процессов, происходящих при отжиге, производилась декомпозиция пика, регистрируемого на дифрактограммах, на два пика: первый пик соответствовал $\kappa_{1}$-фазе, второй пик - ниобату лития и $\alpha$-фазе. Для декомпозиции использовалась функция Гаусса и метод аппроксимации Левенберга-Марквардта. В ходе расчетов проводилось 100 последовательных итераций для более точного соответствия экспериментальных и расчетных кривых. После декомпозиции пика определяли положение максимумов и интегральную интенсивность линий (площадь пика).

Расчеты показали, что значения $\varepsilon_{33}$ для первого пика находятся в пределах (5-7) $10^{-4}$ и соответствуют к Интегральные интенсивности пиков, полученные

Таблица 1. Интегральные интенсивности линий $\left(I^{*}\right)$, соответствующих $\alpha$-фазе и ниобату лития и $\kappa_{1}$-фазе, полученные при декомпозиции отражения (220)

[Table 1. Integrated intensity $\left(I^{*}\right)$ of lines corresponding to $\alpha$ phase and lithium niobate and to $\kappa_{1}$ phase obtained at decomposing (220) reflection]

\begin{tabular}{|c|c|c|}
\hline \multirow{2}{*}{$\begin{array}{c}\text { Продолжительность отжига, ч } \\
\text { [Annealing duration, } \mathrm{h}]\end{array}$} & $\begin{array}{c}|c| \\
\text { [A-фаза и ниобат лития } \\
{[\alpha \text { phase and lithium niobate] }}\end{array}$ & $\begin{array}{c}\kappa_{1} \text {-фаза } \\
{\left[\kappa_{1} \text { phase] }\right.}\end{array}$ \\
\hline 1 & 0.78 & 0.22 \\
\hline 2 & 0.49 & 0.51 \\
\hline 4 & 0.54 & 0.46 \\
\hline 6 & 0.44 & 0.56 \\
\hline 9 & 0.60 & 0.40 \\
\hline
\end{tabular}



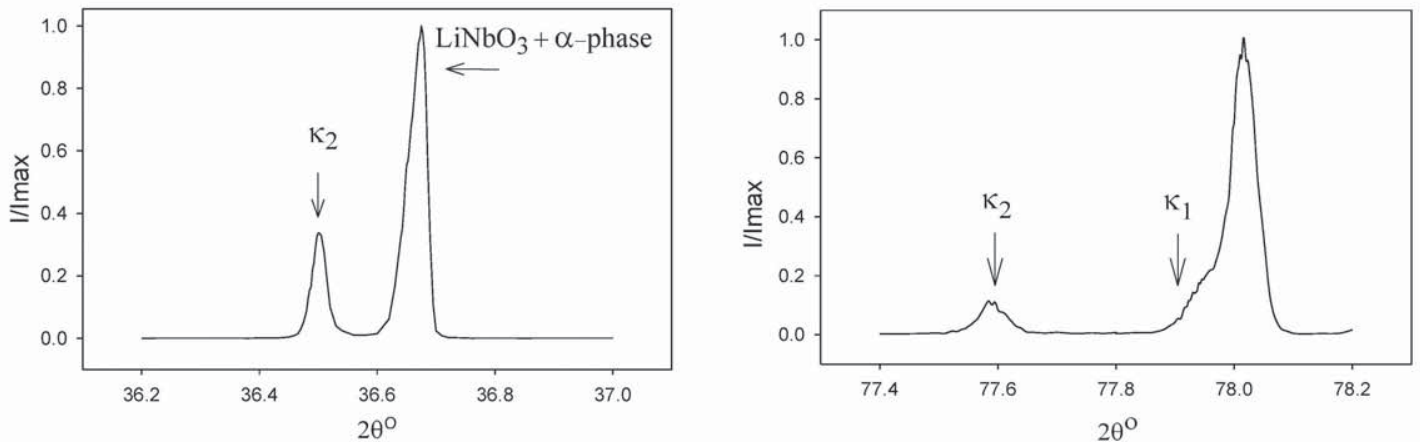

a
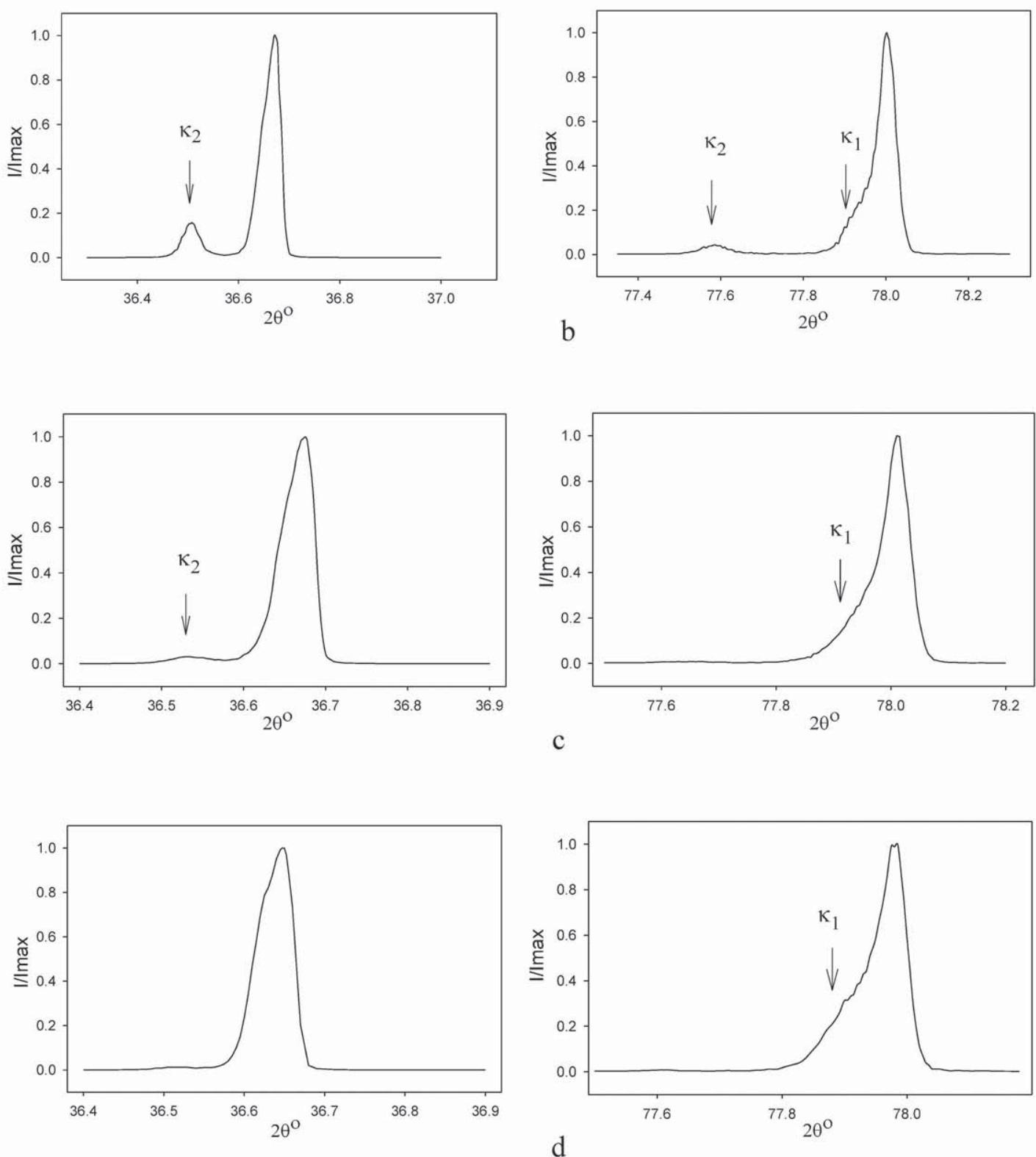

Рис. 4. $\theta / 2 \theta$ кривые протонированных слоев на кристалле ниобата лития после отжига продолжительностью, ч: $a-1, b-2, c-4, d-9$. В левой части рисунка - отражения (110), в правой части - (220)

[Fig. 4. $\theta / 2 \theta$ curves for protonated layers in lithium niobate crystals after annealing of duration, $h$ : $a-1, b-2, c-4, d-9$. At the left - (110) reflections, at the right - (220)] 
при декомпозиции линий (отражение (220)), соответствующих $\alpha$-фазе и ниобату лития и $\kappa_{1}$-фазе, приведены в табл. 1.

Анализируя изменения интегральной интенсивности в процессе отжига, можно сделать следующие заключения о характере изменения фазового состава ПО-слоев: уже после одного часа отжига образуется $\kappa_{1}$-фаза, ее содержание невелико, поскольку в протонообменном слое преобладает к жание $\kappa_{1}$-фазы растет, однако при продолжительности отжига 2-6 ч интегральная интенсивность, соответствующая $\kappa_{1}$-фазе, слабо изменяется, после чего $\left(t>6\right.$ ч) содержание $\kappa_{1}$-фазы уменьшается.

Слабое изменение интегральной интенсивности при отжиге в течение 2-6 ч, возможно, связано с тем, что после 2 ч отжига включения $\kappa_{1}$-фазы незначительно изменяют свои размеры, также слабо изменяется их количество. При этом в процессе отжига происходит диффузия протонов, уменьшается их концентрация в к -фазе. Как было показано в [7], превращение $\kappa_{1}$-фазы в $\alpha$-фазу первоначально происходит при диффузионном контроле процесса. Уменьшение концентрации протонов в $\kappa_{1}$-фазе снижает разницу в показателях преломления $\kappa_{1}$ - и $\alpha$-фаз, из-за чего снижается контраст на оптических микрофотографиях.

В целом, результаты рентгеноструктурного анализа подтверждают следующую последовательность фазовых превращений при отжиге: $\beta_{1}$, $\beta_{2}$-фазы $\rightarrow \kappa_{2}$-фаза $\rightarrow \kappa_{1}$-фаза $\rightarrow \alpha$-фаза. Следует отметить, что второе и третье фазовые превращения в течение определенного времени могут происходить параллельно.

Дополнительную информацию о структурнофазовых превращениях в ПО-слоях дает метод ИКспектроскопии. Непосредственно после протонного обмена в бензойной кислоте протонированные слои состоят из $\beta_{1}^{-}, \beta_{2}$-фаз $[1,9]$. На ИК-спектрах поглощения протонированного ниобата лития (рис. $5 a$ ) наблюдается два пика поглощения: широкий размытый пик с максимумом, соответствующим волновому числу $v \approx 3250 \mathrm{~cm}^{-1}$, и выраженный асимметричный пик с максимумом при $v=3508 \mathrm{~cm}^{-1}$. Согласно [10], размытый пик поглощения соответствует межузельным протонам, принадлежащим $\beta_{2}$-фазе. Поскольку протоны в кристаллической решетке ниобата лития занимают случайные положения, и их энергия связи в кристаллической решетке варьируется в широких пределах, регистрируемый пик находится в широком диапазоне значений $v=3000-3400 \mathrm{~cm}^{-1}$. Второй асимметричный максимум поглощения при $v=3508 \mathrm{~cm}^{-1}$
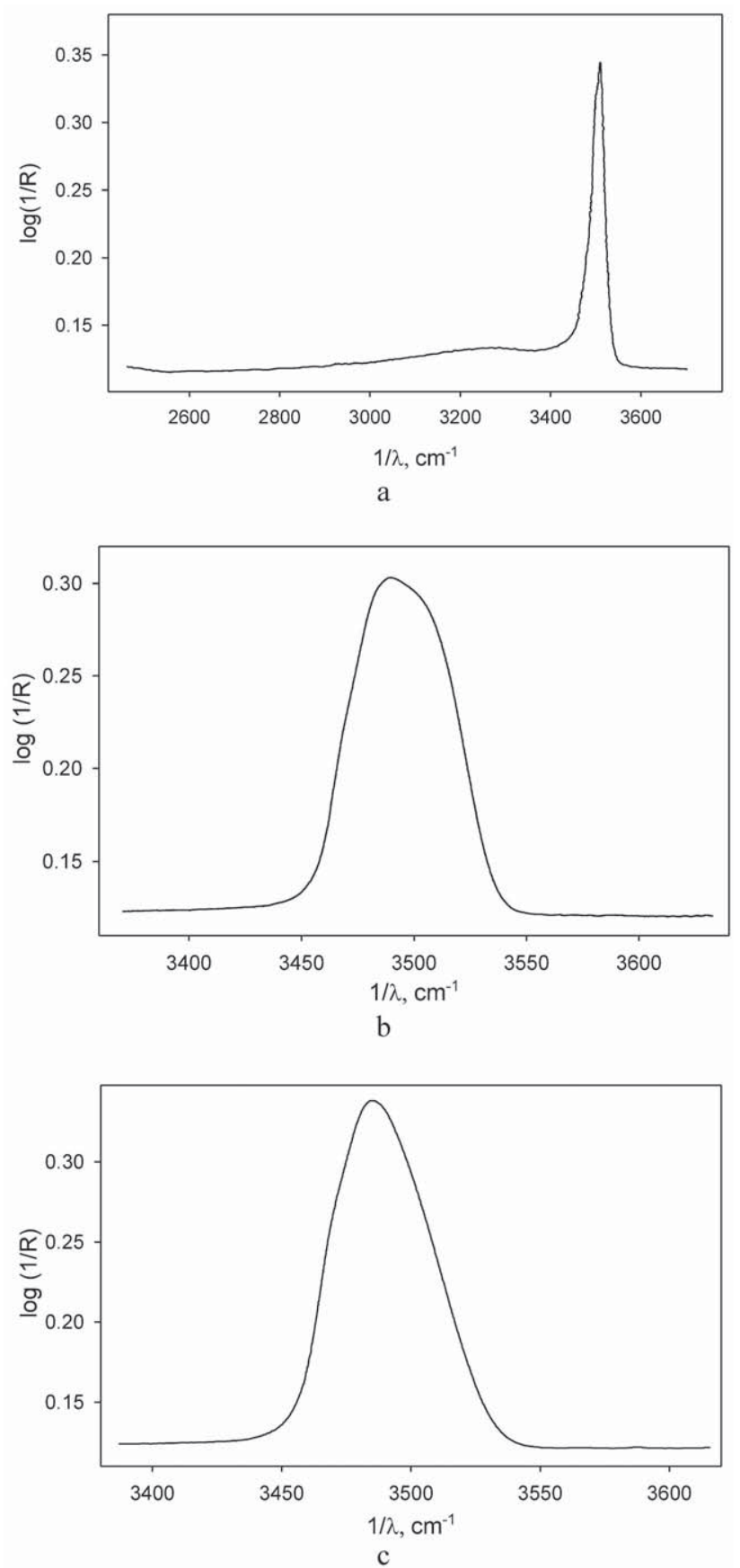

Рис. 5. ИК-спектры поглощения протонированного ниобата лития после отжига в течение, ч:

$$
a-0, b-4, c-11
$$

[Fig. 5. IR absorption spectra of protonated lithium niobate after annealing of duration, h: $a-0, b-4, c-11]$

обусловлен присутствием $\beta_{1}$-фазы и $\alpha$-фазы в протонообменном слое. Декомпозиция данного пика на два пика с использованием функции Гаусса и метода Левенберга-Марквардта дает компоненты с параметрами, приведенными в табл. 2.

Пик со значением волнового числа $3489 \mathrm{~cm}^{-1}$, согласно [10], соответствует $\alpha$-фазе, тогда как пик $3508 \mathrm{~cm}^{-1}$, вероятно, обусловлен присутствием 
Таблица 2. Компоненты полосы поглощения при $~ 3500 \mathrm{~cm}^{-1}$

[Table 2. Components of absorption band at $\sim 3500 \mathrm{~cm}^{-1}$ ]

\begin{tabular}{|c|c|c|c|c|}
\hline $\begin{array}{c}\text { Продолжительность отжига, } \mathrm{u} \\
\text { [Annealing duration, } \mathrm{h}]\end{array}$ & $\begin{array}{c}v_{\text {макс }}, \mathrm{cm}^{-1} \\
{\left[v_{\text {max }}, \mathrm{cm}^{-1}\right]}\end{array}$ & $\begin{array}{c}\text { Высота пика } \\
{[\text { Peak height] }}\end{array}$ & $\begin{array}{c}\text { Полуширина пика, } \mathrm{cm}^{-1} \\
\left.\text { [Peak half-width, } \mathrm{cm}^{-1}\right]\end{array}$ & $\begin{array}{c}\text { Относительная } \\
\text { интенсивность } \\
\text { [Relative intensity] }\end{array}$ \\
\hline 0 & 3508 & 0.17 & 27.2 & 0,48 \\
0.17 & 0.10 & 27.2 & 0.52 \\
\hline 1 & 3489 & 0.15 & 43.8 & 0.28 \\
\hline 4 & 3495 & 0.13 & 30.9 & 0.72 \\
\hline 9 & 3511 & 0.16 & 33.2 & 0.43 \\
\hline 11 & 3486 & 0.20 & 46.1 & 1.00 \\
\hline
\end{tabular}

$\beta_{1}$-фазы в протонообменном слое. В то же время, в работе [10] для $\beta_{1}$-фазы приводится значение $v=3500 \mathrm{~cm}^{-1}$. Однако эти значения были получены в результате протонного обмена на Z-срезе кристалла ниобата лития.

Отжиг в течение 1 ч при температуре $330^{\circ} \mathrm{C}$ приводит к практически полному исчезновению широкого пика, соответствующего $\beta$-фазе, и усилению асимметричности пика при $3500 \mathrm{~cm}^{-1}$. Декомпозиция данного пика дает два пика (табл. 2), вероятно, соответствующих $\kappa_{1}-$ и $\kappa_{2}$-фазам $\left(v=3518 \mathrm{~cm}^{-1}\right)$ и $\alpha$-фазе $\left(v=3495 \mathrm{~cm}^{-1}\right)$.

Дальнейший отжиг (4 ч) усиливает асимметричность пика при $3500 \mathrm{~cm}^{-1}$ (рис. $5 b$ ). При этом, как показали результаты рентгеноструктурных исследований, увеличивается содержание к-фазы и снижается содержание $\kappa_{2}$-фазы в протонообменном слое. Это, возможно, является причиной уменьшения значения волнового числа первого пика. Второй пик, получившийся в результате декомпозиции, достаточно точно соответствует $\alpha$-фазе. Возможно, это связано с упорядочением структуры протонообменного слоя в результате диффузии протонов.

Увеличение продолжительности отжига $\geq 9$ ч (рис. 5c) приводит к тому, что в протонообменном слое присутствует преимущественно $\alpha$-фаза, которой соответствует единственный пик поглощения при 3485-3486 см-1 (табл. 2). Указанные значения соответствуют литературным данным [10]. Это также согласуется с результатами модовой спектроскопии и оптической микроскопии.

Считается, что фазовые превращения в протонообменных слоях при отжиге происходят путем превращения первоначально образовавшихся слоев $\beta_{1}$-и $\beta_{2}$-фаз, когерентно связанных с подложкой ниобата лития, в слои других фаз в зависимости от условий и продолжительности отжига [2].
В данной работе было показано, что при отжиге протонообменных слоев на Х-срезе ниобата лития происходит образование частиц к -фазы. Поскольку для к

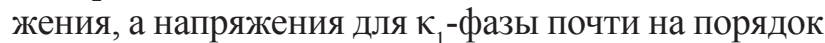
величины меньше, в процессе отжига происходит релаксация напряжений за счет образования некогерентных границ раздела между $\kappa_{2}-$ и $\kappa_{1}$-фазами. Несомненно, образование межфазных границ приводит к образованию дополнительных дефектов структуры. При дальнейшем отжиге даже после исчезновения межфазных границ часть дополнительно образовавшихся дефектов структуры может сохраниться. Эти дефекты структуры могут являться центрами скопления зарядов при изменении температуры, что может оказывать негативное влияние на работу интегрально-оптических устройств, в которых используются протонообменные волноводы.

Следует отметить, что в данной серии экспериментов были получены результаты, в количественном отношении несколько отличающиеся от ранее полученных результатов [7], хотя условия протонного обмена и отжига оставались неизменными. Это связано с тем, что процесс протонного обмена в значительной мере зависит от состояния поверхности кристалла ниобата лития, дефектности его поверхностного слоя $[11,12]$, условий полировки и механической обработки вейферов ниобата лития, а также чистоты используемой бензойной кислоты [13]. Поэтому результаты протонного обмена и последующего отжига на образцах ниобата лития из различных партий могут несколько различаться.

\section{ВЫВОДЫ}

1. С использованием методов модовой спектроскопии, оптической микроскопии в поляризованном свете, рентгеноструктурного анализа и ИК- 
спектроскопии установлена последовательность фазовых превращений при отжиге $\left(330{ }^{\circ} \mathrm{C}\right)$ протоннообменных слоев, сформированных на кристаллах ниобата лития $\left(\mathrm{X}\right.$-срез): $\beta_{1}, \beta_{2}$-фазы $\rightarrow \kappa_{2}$-фаза $\rightarrow \kappa_{1}$-фаза $\rightarrow \alpha$-фаза.

2. Особенностью фазовых превращений на $\mathrm{X}$-срезе кристалла ниобата лития является то, что превращение $\kappa_{2}$-фазы в $\kappa_{1}$-фазу происходит через образование частиц $\kappa_{1}$-фазы (образующих модулированные структуры), а не слоя данной фазы.

Работа выполнена при финансовой поддержке гранта РФФИ (проект № 17-43-590309 р_а).

\section{СПИСОК ЛИТЕРАТУРЫ}

1. Korkishko Yu. N., Fedorov V. A. // IEEE J. Sel. Top. Quantum Electron., 1996, vol. 2, № 2, pp. 187-196.

2. Korkishko Yu. N., Fedorov V. A. // Technical Physics, 1999, vol. 44, № 3, pp. 307-316.

3. Kostritskii S. M., Korkishko Yu. N., Fedorov V. A., Sevostyanov O. G., Chirkova I. M., Mitrokhin V. P. // J. Appl. Spectroscopy, 2015, vol. 82, № 2, pp. 234-241.

4. Suchoski P. G., Findakly T. K., Leonberger F. J. // Optics Letters, 1988, vol. 13, № 11, pp. 1050-1052.
5. Chen S., Baldi P., De Micheli M. P., Ostrowsky D. B., Leycuras A., Tartarini G., Bassi P. // J. Lightwave Technol., 1994, vol. 12, № 5, pp. 862-871.

6. Sun Jian, Xu Chang-qing // J. Appl. Phys., 2015, vol. 117, article ID 043102, 8 pp.

7. Mushinsky S. S., Kichigin V. I., Petukhov I. V., Permyakova M. A., Shevtsov D. I. // Ferroelectrics, 2017, vol. 508, № 1, pp. 40-48.

8. Колосовский Е. А., Петров Д. В., Царев А. В. // Ж. квант. электрон., 1981, т. 8, № 12, с. 2557-2568.

9. Azanova I. S., Shevtsov D. I., Zhundrikov A. V., Kichigin V. I., Petukhov I. V., Volyntsev A. B. // Ferroelectrics, 2008, vol. 374, № 1, pp. 110-121.

10. Christova K., Kuneva M., Tonchev S. // J. Phys.: Conf. Ser., 2010, vol. 253, № 1, article ID 012057, 7 pp.

11. Пономарев Р. С. Структурная модель дрейфовых явлений в интегрально-оптических схемах на основе $\mathrm{H}_{x} \mathrm{Li}_{1-x} \mathrm{NbO}_{3}$ канальных волноводов. Дисс. ... канд. физ.мат. наук. Пермь, 2014. 148 с.

12. Sosunov A. V., Ponomarev R. S., Mushinsky S. S., Minkin A. M., Volyntsev A. B. // Ferroelectrics, 2016, vol. 494, № 1, pp. 131-137.

13. Петухов И. В., Кичигин В. И., Мушинский С. С., Минкин А. М., Шевцов Д. И. // Конденсированныле среды и межфазные границы, 2012, т. 14, № 1, c. $119-123$.

\title{
STRUCTURAL PHASE TRANSITIONS IN PROTON EXCHANGED LAYERS OF LITHIUM NIOBATE CRYSTALS DURING ANNEALING
}

\author{
(C) 2017 S. S. Mushinsky, I. V. Petukhov, M. A. Permyakova, V. I. Kichigin, \\ L. N. Malinina, A. B. Volyntsev \\ Perm State University, 15 Bukirev str., Perm, Russia \\ e-mail: Petukhov-309@yandex.ru
}

Received 04.08.2017

\begin{abstract}
Lithium niobate crystals are widely used in the manufacturing of integrated optical phase and intensity modulators. The production method is based on the formation of optical waveguides in the surface layers of $\mathrm{LiNbO}_{3}$ by means of proton exchange. Subsequent annealing of proton-exchanged structures is usually performed in order to obtain stable and low-loss waveguides.

The aim of this paper is to study phase transitions in the surface layer of proton-exchanged waveguides on X-cuts of lithium niobate crystals during annealing. In our research, the following methods were used: polarized light optical microscopy (bright field and dark field), mode spectroscopy (determination of the refractive index profile), IR-spectroscopy, and XRD ( $\theta / 2 \theta$ curves). Congruent lithium niobate (Crystal Technology) was used as a material. Proton exchange was carried out in molten benzoic acid at $175{ }^{\circ} \mathrm{C}$ for 2 hours. The samples were then annealed at 1-hour intervals at $330{ }^{\circ} \mathrm{C}$. The total duration of annealing was 12 hours.

$\beta_{1}$ and $\beta_{2}$ phases formed during the proton exchange stage, transformed into the $\kappa_{2}$-phase within the first hour of annealing. After the first annealing step ( 1 hour) small particles of the $\kappa_{1}$-phase appeared in the matrix of the $\kappa_{2}$-phase. The $\kappa_{2}$-phase gradually transformed into the $\kappa_{1}$-phase within the 2 nd and 3rd hour of annealing. $\kappa_{1}$-phase particles formed a modulated structure along certain crystallographic directions. The $\kappa_{1}$-phase then slowly transformed into $\alpha$-phase. Residues of the $\kappa_{1}$-phase were still detected by means of optical microscopy after up to 10 hours of annealing. The structure became fully homogeneous after 12 hours of annealing. The results obtained previously by various methods comply with this phase transition sequence.
\end{abstract}


The formation of $\kappa_{1}$-phase particles can be explained by the significant difference in internal stresses of the $\kappa_{2}$ and $\kappa_{1}$ phases formed on X-cuts of lithium niobate. During the annealing, relaxation takes place due to the incoherence of the boundary between the $\kappa_{2}$ - and $\kappa_{1}$-phases.

Keywords: lithium niobate, X-cut, proton exchange, annealing, phase transformation.

\section{ACKNOWLEDGEMENTS}

The reported study was supported by the Russian Foundation for Basic Research (project No. 17-43590309 r_a).

\section{REFERENCES}

1. Korkishko Yu. N., Fedorov V. A. IEEE J. Sel. Top. Quantum Electron., 1996, vol. 2, no. 2, pp. 187-196. DOI: $10.1109 / 2944.577359$

2. Korkishko Yu. N., Fedorov V. A. Technical Physics, 1999, vol. 44, no. 3, pp. 307-316. DOI: 10.1134/1.1259243

3. Kostritskii S. M., Korkishko Yu. N., Fedorov V. A., Sevostyanov O. G., Chirkova I. M., Mitrokhin V. P. J. Appl. Spectroscopy, 2015, vol. 82, no. 2, pp. 234-241. DOI: 10.1007/s10812-015-0091-2

4. Suchoski P. G., Findakly T. K., Leonberger F. J. Optics Letters, 1988, vol. 13, no. 11, pp. 1050-1052. DOI: 10.1364/OL.13.001050

5. Chen S., Baldi P., De Micheli M. P., Ostrowsky D. B., Leycuras A., Tartarini G., Bassi P. J. Lightwave Technol., 1994, vol. 12, no. 5, pp. 862-871. DOI: 10.1109/50.293979

6. Sun Jian, Xu Chang-qing. J. Appl. Phys., 2015, vol. 117, article ID 043102, 8 pp. DOI: $10.1063 / 1.4906222$
7. Mushinsky S. S., Kichigin V. I., Petukhov I. V., Permyakova M.A., Shevtsov D. I. Ferroelectrics, 2017, vol. 508, no. 1, pp. 40-48. DOI: 10.1080/00150193.2017.1286702

8. Kolosovskii E. A., Petrov D. V., Tsarev A. V. Sov. J. Quantum Electron., 1981, vol. 11, no. 12, pp. 1560-1566. DOI: 10.1070/QE1981v011n12ABEH008650

9. Azanova I. S., Shevtsov D. I., Zhundrikov A. V., Kichigin V. I., Petukhov I. V., Volyntsev A. B. Ferroelectrics, 2008, vol. 374, no. 1, pp. 110-121. DOI: 10.1080/0015019 0802427234

10. Christova K., Kuneva M., Tonchev S. J. Phys.: Conf. Ser., 2010, vol. 253, no. 1, article ID 012057, 7 pp. DOI: 10.1088/1742-6596/253/1/012057

11. Ponomarev R. S. A Structural Model for Drift Phenomena in Integrated Optical Circuits Based on $\mathrm{H}_{\mathrm{Li}} \mathrm{NbO}_{3}$ Channel Waveguides. Diss. ... cand. phys.-math. sci. Perm, 2014. 148 p. (in Russian).

12. Sosunov A. V., Ponomarev R. S., Mushinsky S. S., Minkin A. M., Volyntsev A. B. Ferroelectrics, 2016, vol. 494, no. 1, pp. 131-137.

13. Petukhov I. V., Kichigin V. I., Mushinsky S. S., Minkin A. M., Shevtsov D. I. Condensed Matter and Interphases, 2012, vol. 14, no. 1, pp. 119-123. Available at: http://www.kcmf.vsu.ru/resources/t_14_1_2012_019.pdf
Мушинский Сергей Сергеевич - инженер, Пермский государственный национальный исследовательский университет; тел.: +7(342) 2396789, e-mail: sergey.mushinsky@gmail.com

Петухов Игорь Валентинович - к. х. н., доцент кафедры физической химии, Пермский государственный национальный исследовательский университет; тел.: +7(342) 2396789, e-mail: petukhov-309@yandex.ru

Пермякова Мария Александровна - магистрант кафедры физической химии, Пермский государственный национальный исследовательский университет; тел.: +7(342) 2396789

Кичигин Владимир Иванович - к. х. н., с. н. с. кафедры физической химии, Пермский государственный национальный исследовательский университет; тел.: +7(342) 2396452, e-mail: kichigin@psu.ru

Малинина Людмила Николаевна - инженер кафедры физики твердого тела, Пермский государственный национальный исследовательский университет; тел.: $+7(342) 2396383$

Вольниев Анатолий Борисович - д. ф.-м. н., профессор, заведующий кафедрой физики твердого тела, Пермский государственный национальный исследовательский университет; тел.: +7(342) 2396410, e-mail: voland@psu.ru
Myshinsky Sergey S. - Engineer, Perm State University, ph.: +7(342) 2396789, e-mail: sergey.mushinsky@gmail. com

Petukhov Igor V. - Cand. Sci. (Chem.), Associate Professor, Department of Physical Chemistry, Perm State University; ph.:+7(342) 2396789, e-mail: petukhov-309@ yandex.ru

Permyakova Mariya A. - graduate student, Department of Physical Chemistry, Perm State University; ph.: +7(342) 2396789

Kichigin Vladimir I. - Cand. Sci. (Chem.), Senior Researcher, Department of Physical Chemistry, Perm State University; ph.: +7(342) 2396452, e-mail: kichigin@psu. ru

Malinina Ljudmila N. - Engineer of Solid State Physics Department, Perm State University; ph.: +7(342) 2396383

Volyntsev Anatoliy B. - Dr. Sci. (Phys.-Math.), Professor, Head of Solid State Physics Department, Perm State University; ph.: +7(342) 2396410, e-mail: voland@psu.ru 